\title{
Observational evidence for mass ejection during soft X-ray dips in GRS 1915+105
}

\author{
S. V. Vadawale ${ }^{1}$, A. R. Rao ${ }^{1}$, A. Nandi ${ }^{2}$, and S. K. Chakrabarti ${ }^{2}$ \\ 1 Tata Institute of Fundamental Research, Homi Bhabha Road, Mumbai (Bombay) 400 005, India \\ 2 S.N. Bose National Center for Basic Sciences, Salt Lake, Calcutta 700 091, India
}

Received 8 January 2001 / Accepted 1 March 2001

\begin{abstract}
We investigate the connection between the X-ray and radio properties of the Galactic microquasar GRS 1915+105, by analyzing the X-ray data observed with RXTE, during the presence of a huge radio flare $(\sim 450 \mathrm{mJy})$. The X-ray lightcurve shows two dips of $\sim 100 \mathrm{~s}$ duration. Detailed time resolved spectral analysis shows the existence of three spectral components: a multicolor disk-blackbody, a Comptonized component due to hot plasma and a power-law. We find that the Comptonized component is very weak during the dip. This is further confirmed by the PHA ratio of the raw data and ratio of the lightcurves in different energy bands. These results, combined with the fact that the $0.5-10 \mathrm{~Hz}$ QPO disappears during the dip and that the Comptonized component is responsible for the QPO lead to the conclusion that during the dips the matter emitting Comptonized spectrum is ejected away. This establishes a direct connection between the X-ray and radio properties of the source.
\end{abstract}

Key words. accretion, accretion disks - black hole physics - stars: winds, outflows - stars: individual: GRS 1915+105 - X-rays: stars

\section{Introduction}

The Galactic microquasar GRS $1915+105$ is a bright $\mathrm{X}$-ray source and it is a subject of intense study in all wavelengths, particularly in radio and X-ray wavelengths (see Mirabel \& Rodriguez 1999 and references therein). It has been exhibiting different types of X-ray variability characteristics (Morgan et al. 1997; Muno et al. 1999; Yadav et al. 1999; Belloni et al. 2000a). The radio emission from this source also demonstrates its chaotic nature by means of time to time huge radio flares (Mirabel \& Rodriguez 1994; Fender et al. 1999), long episodes of high/low emissions and periodic oscillations (Pooley \& Fender 1997). There were several attempts in the past to correlate the radio and X-ray emission characteristics. Pooley \& Fender (1997) reported short period radio oscillations coincident with X-ray dips. Fender \& Pooley (1998) showed that the IR emission, interpreted as the high-energy tail of a synchrotron spectrum, also varies on similar time scales. Feroci et al. (1999) reported disappearance of inner accretion disk during a small radio flare. Thus, so far there are many evidences for the morphological correlation between X-ray emission and small radio oscillations or flares. However, in the case of huge radio flares, exhibited by this source from time to time, there is no strong morphological identification with

Send offprint requests to: S. V. Vadawale, e-mail: santoshv@tifr.res.in detailed X-ray emission characteristics. Fender et al. (1999) suggested that the repeated ejections of the inner accretion disk (Belloni et al. 1997) might be responsible for such flares. It was pointed out that such oscillations, having hard dips are not always accompanied by high radio emission (Naik \& Rao 2000; Yadav et al. 1999). This suggests that some other mechanism is responsible for such huge radio flares. Recently, Naik \& Rao (2000) made a systematic study of the morphology of different types of $\mathrm{X}$-ray emission and accompanying radio emission and found an one to one correspondence between the soft dips in X-rays (observed during classes $\beta$ and $\theta$ ) and high radio emission. Naik et al. (2001) have suggested that the huge radio flares might be produced due to a number of such soft dip events.

In this Letter we propose an evidence of mass ejection, during the soft X-ray dips, by performing a detailed time resolved X-ray spectroscopy of the RXTE archival data observed simultaneously with a huge radio flare. We identify three components in the spectrum and show that the Comptonized component disappears during the dips. We explain this as ejection of the inner cloud and thus establish a direct connection between huge radio flares and $\mathrm{X}$-ray emission from this source.

\section{Analysis and results}

GRS 1915+105 exhibits huge radio flares from time to time, the most recent of which occurred on 1999 June 8. 


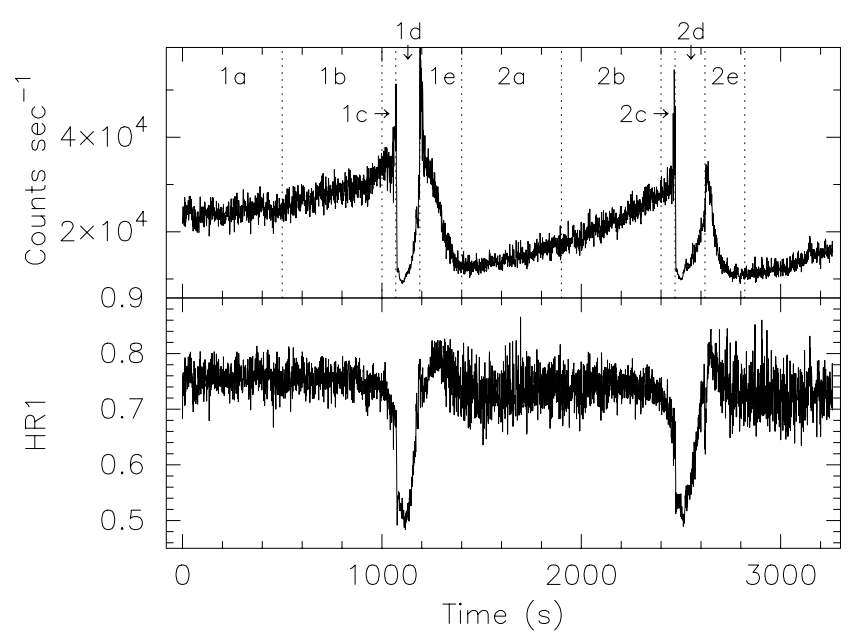

Fig. 1. Lightcurve (top panel) and hardness ratio (6-15 keV/2-6 keV; bottom panel) of GRS 1915+105 obtained on 1999 June 8 using $R X T E-P C A$. The regions chosen for time resolved spectral and temporal studies are shown in the top panel, separated by dotted lines

The PPC detectors of IXAE (Agrawal et al. 1997) observed the source during the entire episode of this radio flare, including the low-hard state of the source just prior to the flare. The IXAE observations revealed the presence of regular soft dips in the X-ray lightcurve during the radio flare. During these dips the X-ray flux decreases by a factor of three within $\sim 5 \mathrm{~s}$, remains low for $\sim 30-60 \mathrm{~s}$ and then gradually recovers to the maximum (Naik et al. 2001). Inspired by this observation, we obtained the RXTE data observed on 1999 June 8 (ObsID: 40702-01-03-00) to study, in detail, the spectral properties of the dips during the radio flare. This is the only pointed RXTE observation during this flare (Naik et al. 2001). The PCA (Jahoda et al. 1996) lightcurve and hardness ratio for this observation is shown in Fig. 1. It shows that this observation belongs to class $\theta$ as defined by Belloni et al. (2000a). The class $\theta$ shows almost regular soft dips of 40-100 s duration (defined as state A) and variable lowhard state (defined as state C) outside the dip.

We have attempted a wide band, time-resolved X-ray spectroscopy of the dip events by making spectral fits to the data from different portions of the lightcurve during both the observed cycles. We have divided each cycle into five intervals: pre-pre-dip (a, $500 \mathrm{~s}$ ), pre-dip (b, $500 \mathrm{~s}$ ), edge (c, $80 \mathrm{~s})$, dip (d, $100 \mathrm{~s}$ and $140 \mathrm{~s})$ and post-dip (e, $200 \mathrm{~s}$ ). Figure 1 (top panel) shows the selection of these time intervals. For wide band spectral fitting we have extracted 129 channel spectra from PCA and 64 channel spectra from HEXTE. We have used data from cluster 0 of HEXTE and have added $2 \%$ systematic error to PCA spectra (Vadawale et al. 2001; Gierlinski et al. 1999). The spectra during very short intervals e.g. dip and edge, were rebinned to fewer number of channels in order to improve the statistics. We have fitted the PCA $(3-50 \mathrm{keV})$ and

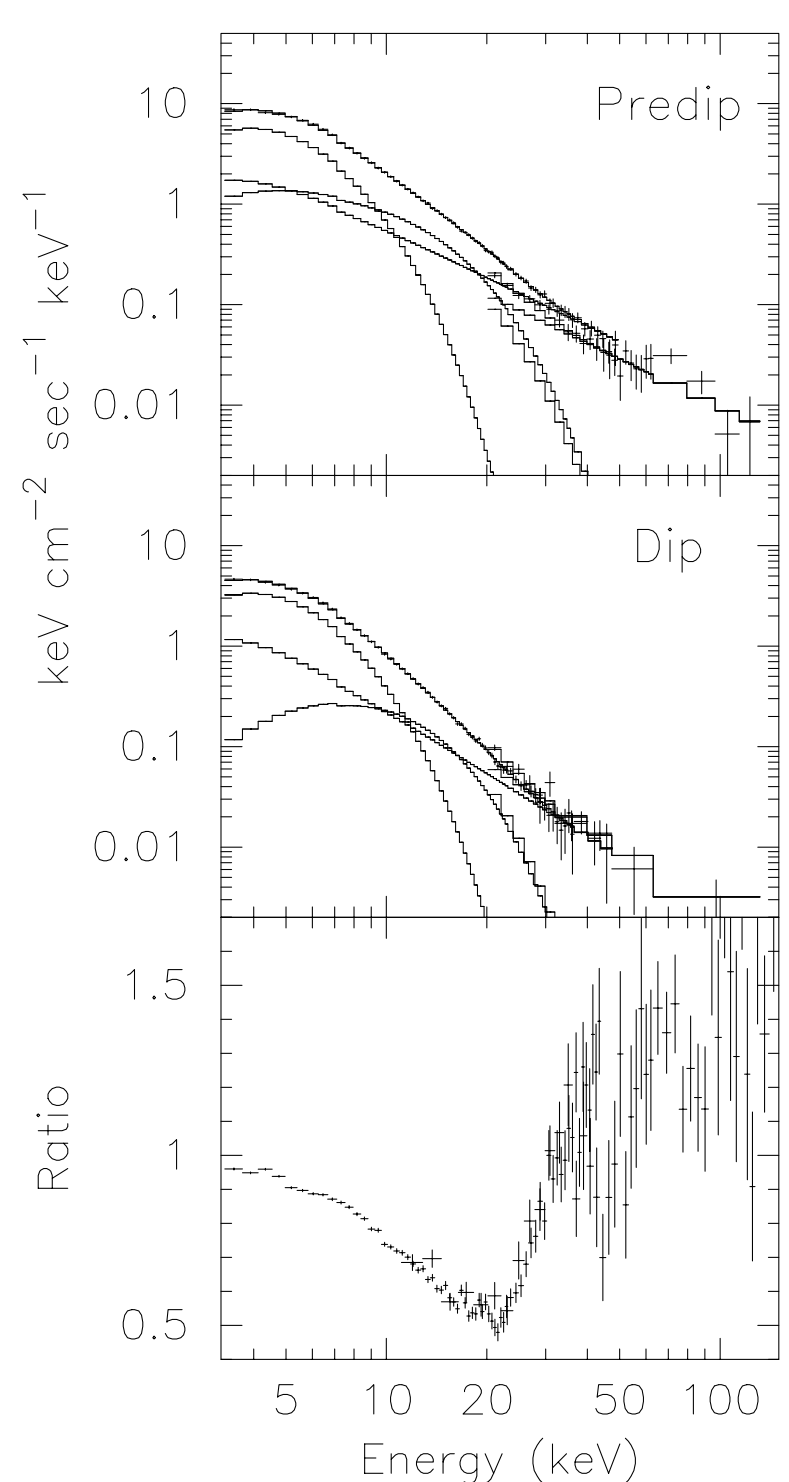

Fig. 2. The deconvolved X-ray spectra of GRS 1915+105 during the last $80 \mathrm{~s}$ before the dip (top panel) and $100 \mathrm{~s}$ during the dip (middle panel). The model consists of a disk blackbody, Comptonization due to hot plasma, and a power-law. The bottom panel shows the ratio of observed count rates (dip to pre-dip ratio) normalized at $30 \mathrm{keV}$, which highlights the lack of counts in the range $8-30 \mathrm{keV}$ during the dip

HEXTE (15-150 keV) spectra simultaneously with different models (see Vadawale et al. 2001; Rao et al. 2000).

We find that the X-ray spectrum cannot be fitted by the "standard" model for the Black Hole Candidates (BHCs), consisting of a disk-blackbody and a power-law. It is also known previously that the X-ray spectra of radio loud states are peculiar and cannot be described by the standard model (Muno et al. 1999; Belloni et al. 2000b) and hence it is necessary to look for more complex models. We find that a three component model consisting of a disk-blackbody, a Comptonization due to hot plasma (CompST - see Sunyaev \& Titarchuk 1980) and a powerlaw is necessary for statistically and physically acceptable fit to the X-ray spectra of the current observation. 
Table 1. Results of temporal and spectral analysis ${ }^{1}$ of RXTE data of GRS $1915+105$ during class $\theta$ on 1999 June 8

\begin{tabular}{|c|c|c|c|c|c|c|c|c|c|c|}
\hline \multirow[t]{2}{*}{ Region } & \multirow{2}{*}{$\begin{array}{c}\nu_{\mathrm{QPO}} \\
\mathrm{Hz}\end{array}$} & \multirow{2}{*}{$\begin{array}{c}P_{\mathrm{QPO}} \\
\mathrm{rms} \%\end{array}$} & \multirow{2}{*}{$\begin{array}{c}k T_{\text {in }} \\
(\mathrm{keV})\end{array}$} & \multirow{2}{*}{$\begin{array}{c}k T_{\mathrm{e}} \\
(\mathrm{keV})\end{array}$} & \multirow[t]{2}{*}{$\tau$} & \multirow[t]{2}{*}{$\Gamma_{\mathrm{X}}$} & \multicolumn{4}{|c|}{$2-50 \mathrm{keV}$ Flux $\left(10^{-8} \mathrm{erg} \mathrm{cm}^{-2} \mathrm{~s}^{-1}\right)$} \\
\hline & & & & & & & Total & Diskbb & CompST & Power-law \\
\hline $1 \mathrm{a}$ & 5.99 & 3.10 & 1.51 & 3.35 & $\begin{array}{l}16.77 \\
\end{array}$ & 2.81 & 6.69 & 2.00 & 1.13 & 3.56 \\
\hline $1 b$ & 6.68 & 2.47 & 1.58 & 3.55 & 12.92 & 2.86 & 7.71 & 2.46 & 1.55 & 3.70 \\
\hline $1 \mathrm{c}$ & 8.34 & 1.89 & 1.51 & 3.18 & 12.85 & 2.61 & 8.80 & 4.25 & 2.20 & 2.34 \\
\hline $1 d$ & - & - & 1.50 & 2.58 & 36.28 & 3.11 & 4.07 & 2.49 & 0.45 & 1.12 \\
\hline $1 \mathrm{e}$ & 4.67 & 3.44 & 1.56 & 3.52 & 12.86 & 2.75 & 6.27 & 2.12 & 1.28 & 2.86 \\
\hline $2 \mathrm{a}$ & 4.71 & 6.74 & 1.36 & 3.60 & 13.69 & 2.74 & 4.17 & 0.98 & 0.68 & 2.50 \\
\hline $2 \mathrm{~b}$ & 5.60 & 3.76 & 1.50 & 3.64 & 12.75 & 2.84 & 6.18 & 1.66 & 1.08 & 3.44 \\
\hline $2 \mathrm{c}$ & 7.27 & 1.65 & 1.45 & 3.14 & 21.53 & 2.60 & 7.52 & 3.66 & 1.90 & 1.96 \\
\hline $2 \mathrm{~d}$ & - & - & 1.44 & 2.40 & 35.30 & 2.93 & 3.95 & 2.35 & 0.53 & 1.06 \\
\hline $2 \mathrm{e}$ & 4.20 & 6.57 & 1.42 & 3.52 & 13.66 & 2.61 & 4.44 & 1.42 & 0.96 & 2.06 \\
\hline
\end{tabular}

${ }^{1}$ The model components are disk blackbody (diskbb), thermal-Compton (CompST) and power-law. Typical errors: inner disk temperature $k T_{\text {in }}: \pm 0.03$; temperature of the Compton cloud $k T_{\mathrm{e}}: \pm 0.15$; optical depth of the Compton cloud $\tau: \pm 0.40$; power-law photon index $\Gamma_{\mathrm{X}}: \pm 0.05$;

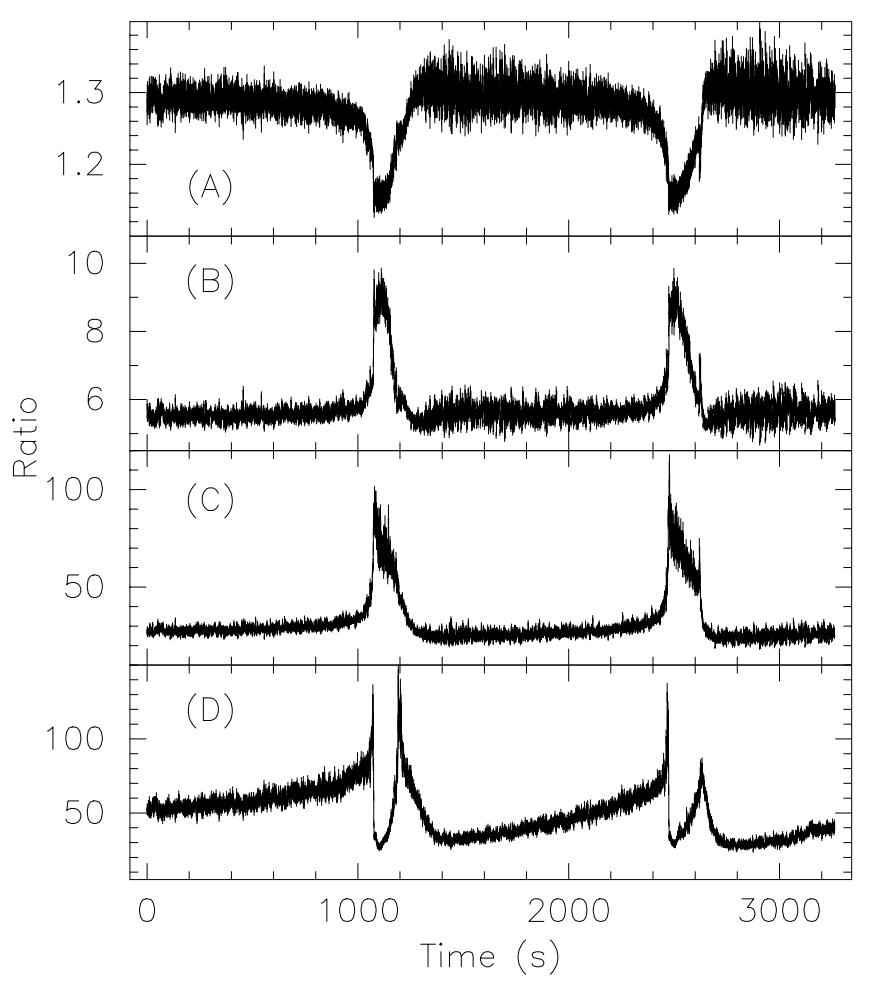

Fig. 3. Ratio of the lightcurves of GRS $1915+105$ a) std- $1 / 2$ $8 \mathrm{keV} \mathrm{b})$ std- $1 / 8-15 \mathrm{keV}$ c) std- $1 / 15-25 \mathrm{keV}$ d) std- $1 / 25$ $60 \mathrm{keV}$. Opposite shape of the dip in the top/bottom and middle two panels suggests that during the dip, relative decrease of counts in the middle energy ranges is larger than that in the low/high energy range

For example, in the pre-dip1 region, a model consisting of a disk-blackbody and a power-law gives $\chi^{2}=272$ for 89 degrees of freedom (dof), a model consisting of a diskblackbody and a CompST gives $\chi^{2}=191$ for 88 dof, whereas the model consisting of three component: a diskblackbody, a CompST and a power-law gives $\chi^{2}=85$ for 86 dof. The spectral fits improve by similar orders in the other regions as well, by using the three component model. The same model is used by Vadawale et al. (2001) and Rao et al. (2000) to describe the radio loud low-hard state of this source, and they give a detailed justification for the existence of the third component. It should be mentioned here that the wide band (3-150 keV) spectral fitting is critical for identification of all the three components. Vadawale et al. (2001) have suggested the origin of the additional power-law as the very high-energy tail of the synchrotron radiation responsible for the radio emission. Our results strengthen their conclusion that the high radio emission manifests itself in X-ray spectra as an additional power-law component. The parameters of the best fit model are shown in Table 1 along with the componentwise X-ray flux for each region separately. The observed values of $\mathrm{QPO}$ frequency and the rms power in the QPO are also given in the table.

The two dip periods are particularly interesting because of the very week CompST component and the absence of QPO. The absence of QPO (also reported earlier by Muno et al. 1999; Markwardt et al. 1999), combining with the result that only the CompST is responsible for the QPO (Rao et al. 2000), suggests that the CompST should also be absent during the dips. The same is indicated by the large decrease in the CompST flux compared to other two component fluxes (Table 1). A small CompST flux in the dip could be due to the inclusion of the recovery period in the dip spectrum. It is not possible to get the combined PCA and HEXTE-CL0 spectra during the first $\sim 60$ s of dip minima due to the rocking motion of the HEXTE clusters. However, spectral analysis of the dip minima using only PCA data shows that a diskblackbody + power-law model gives statistically acceptable fit $\left(\chi^{2}=84\right.$ for 60 dof $)$, whereas the same model, for the pre-dip1 PCA data, gives unacceptable fit $\left(\chi^{2}=220\right.$ for 64 dof). This leads to a hypothesis that the CompST is really absent in the beginning of the dip and slowly reappears during the later part of the dip.

To verify this hypothesis, we examined various ratios of the raw data. First two panels of Fig. 2 show the 
unfolded spectra of pre-dip and dip intervals, obtained for the first dip, whereas the bottom panel shows the ratio of the observed count rate during the dip period to that during the pre-dip period, normalized at $30 \mathrm{keV}$. This ratio clearly shows that the dip period has fewer counts in the middle energy range $(10-30 \mathrm{keV})$, in which the spectrum is dominated by CompST component, compared to the low and high energy range. This justifies our hypothesis that only CompST vanishes during the dip. To examine the temporal behavior of the dip in different energy ranges, we show in Fig. 3, the ratio of the PCA Standard1 lightcurve (consisting of photons of all energy) to the lightcurve in different energy ranges. First panel of this figure shows that, during the dip, decrease in the count-rate in $2-8 \mathrm{keV}$ range is less then that in the total count-rate, whereas the second and third panel show that, during the dip, decrease in the count-rate in $8-25 \mathrm{keV}$ range (where CompST is a dominant component) is more than that in the total count-rate. Opposite shape of the dip in the first two panels shows that the decrease in the count-rate during the dip is strongly energy dependent and is most in the range where CompST is dominating. The shape of the dips in the fourth panel, which are shallower than the dips in the total lightcurve and thus show the effect of the dips in the lightcurve above $25 \mathrm{keV}$, is also opposite to that in the third panel. This provides further evidence to our hypothesis by showing that the decrease in the count-rate at high energies, is less than that in the middle energies.

Thus Figs. 2 and 3 provide strong support to our hypothesis, made from the time resolved spectroscopy, that the CompST component disappears during the dips. This can be interpreted as the ejection of the matter of the Compton cloud.

\section{Discussion}

Microquasars are thought to be the Galactic analogues of the distant quasars and AGNs. Because of their very low mass, compared to the AGNs, they provide a unique opportunity to probe the astrophysics of the AGNs in very short time scales. GRS $1915+105$ is one of the most active microquasars, and shows all possible modes of the mass inflow and outflow, exhibited by means of highly complex emission throughout the electromagnetic band. Belloni et al. (2000a) classified the different types of the $\mathrm{X}$-ray emission from this source in 12 different classes and the present observation belongs to the class $\theta$, which predominantly consists of the low-hard state and soft dips. Our finding, that the X-ray spectrum of the low-hard state outside the dips in this observation is best described by three component model corroborates the conclusion drawn by Vadawale et al. (2001), who found that the X-ray spectra of all radio-loud low-hard states require the three component model.

The vanishing of the Comptonized component during the dip leads to the interpretation that the matter responsible for the Comptonized component is ejected away from the inner region of the accretion disk and the ejected matter emits the synchrotron radiation which is observed as the radio flare. As time progresses, this matter is replenished and the Comptonized component reappears. Nandi et al. (2001) estimate a mass of $\sim 10^{18} \mathrm{~g}$ to be ejected during a dip event, based on the TCAF model, and they give a physical basis for such an ejection. The radio flare which occurred on 1999 June 8 is fairly large, with flux at $2.25 \mathrm{GHz}$ reaching up to $500 \mathrm{mJy}$, which are previously observed only during the superluminal ejection from this source. Rodriguez \& Mirabel (1999) have estimated a typical mass of the superluminal ejecta of the order of $10^{22}-10^{23} \mathrm{~g}$ and hence a collection of a large number of such dips can cause the ejection of the superluminal ejecta. These results give a concrete support to the suggestion made by Naik et al. (2001) that the huge radio flares are produced by multiple dip events.

We wish to point out here that the hard state (state C) outside the soft dips, and variations in them, are also associated with radio emission in GRS 1915+105. Yadav (2001) has found a correlation between the X-ray hardness ratio in state $\mathrm{C}$ and the strength of radio emission for various X-ray variability classes. Belloni et al. (2000b) have estimated the mass accretion rate from the changes in the sizes of the inner accretion disk and have associated them with the outflow rates. In this work, we have found a definite evidence from X-ray spectroscopic analysis for a particular emission region disappearing during the soft dips (state A). It is quite possible that the state $\mathrm{C}$ (and variations in them) is associated with flat spectrum radio emission (see also Vadawale et al. 2001) and the soft dips are associated with steep spectrum radio emission coming from the superluminally moving ejecta. A continuous $\mathrm{X}$-ray and radio observations during a superluminal jet emission episode should throw further light on the origin of radio emission in GRS 1915+105.

\section{Conclusion}

In this Letter we have shown that during soft X-ray dips, decrease in the count rate in the middle energy range $(8-25 \mathrm{keV})$ is significantly greater than that in the low/high energies. We explain this as an ejection of a Compton cloud, whose radiation dominates in the middle energy range. We suggest that a collection of a large number of such dips can eject the required mass of the superluminally moving blobs.

Acknowledgements. We thank referee for useful comments and suggestions to improve the presentation of this paper. This research has made use of data obtained through the High Energy Astrophysics Science Archive Research Center Online Service, provided by the NASA/Goddard Space Flight Center.

\section{References}

Agrawal, P. C., et al. 1997, J. Korean Astrono. Soc., 29, S429 Belloni, T., Klein-Wolt, M., Mendez, M., van der Klis, M., \& van Paradijs, J. 2000a, A\&A, 355, 271

Belloni, T., Migliari, S., \& Fender, R. P. 2000b, A\&A, 358, L29 
Belloni, T., Mendez, M., King, A. R., van der Klis, M., \& van Paradijs, J. 1997, ApJ, 479, L145

Chakrabarti, S. K., \& Titarchuk, L. G. 1995, ApJ, 455, 623

Fender, R. P., \& Pooley, G. G. 1998, MNRAS, 300, 573

Fender, R. P., Garrington, S. T., McKay, D. J., et al. 1999, MNRAS, 304, 865

Feroci, M., Matt, G., Pooley, G., et al. 1999, A\&A, 351, 985

Gierlinski, M., Zdziarski, A. A., Poutanen, J., \& Coppi, P. S. 1999, MNRAS, 309, 496

Jahoda, K., et al. 1996, SPIE, 2808, 59

Markwardt, C. B., Swank, J. H., \& Taam, R. E. 1999, ApJ, 513, L37

Mirabel, I. F., \& Rodriguez, L. F. 1994, Nature, 371, 46

Mirabel, I. F., \& Rodriguez, L. F. 1999, ARA\&A, 37, 409

Morgan, E. H., Remillard, R. A., \& Greiner, J. 1997, ApJ, 482, 993

Muno, M. P., Morgan, E. H., \& Remillard, R. A. 1999, ApJ,
527,321

Naik, S., \& Rao, A. R. 2000, A\&A, 362, 691

Naik, S., Agrawal, P. C., Rao, A. R., et al. 2001, ApJ, 546, 1075

Nandi, A., Chakrabarti, S. K., Vadawale, S. V., \& Rao, A. R. 2001, A\&A, submitted

Pooley, G. G., \& Fender, R. P. 1997, MNRAS, 292, 925

Rao, A. R., Naik, S., Vadawale, S. V., \& Chakrabarti, S. K. 2000, A\&A, 360, L25

Rodriguez, L. F., \& Mirabel, I. F. 1999, ApJ, 511, 398

Vadawale, S. V., Rao, A. R., \& Chakrabarti, S. K. 2001, A\&A, submitted

Sunyaev, E. A., \& Titarchuk, L. 1980, A\&A, 86, 121

Yadav, J. S., Rao, A. R., Agrawal, P. C., et al. 1999, ApJ, 517, 935

Yadav, J. S. 2001, ApJ, 548, 876 